\title{
On the Performance of Hybrid Functionals for Non-linear Optical Properties and Electronic Excitations in Chiral Molecular Crystals: The Case of Butterfly-Shaped Dicinnamalacetone
}

\author{
Pedro S. Pereira da Silva, ${ }^{*[a]}$ Pablo Martín-Ramos, ${ }^{[a, b]}$ Sérgio R. Domingos, ${ }^{[c]}$ \\ Maria do Carmo Bota de Sousa, ${ }^{[\mathrm{d}]}$ Cláudia T. Arranja, ${ }^{[\mathrm{d}]}$ Abílio J. F. N. Sobral, ${ }^{[\mathrm{d}]}$ and \\ Manuela Ramos Silva ${ }^{[a]}$
}

\begin{abstract}
Purely organic chiral molecular assemblies in the solid state hold great potential for non-linear optical applications. Herein, a newly synthesised molecular system is reported, namely, dicinnamalacetone, an otherwise planar molecule that crystallises in a disordered non-centrosymmetric form with four different conformations having an overall predominance of a particular helicity. A combined experimental and theoretical ap-
\end{abstract}

proach, including single-crystal X-ray diffraction, Kurtz-Perry and $a b$ initio methods, is employed to characterise the system and benchmark the performance of hybrid functionals for the prediction of non-linear optical properties and electronic excitations. Comparison of experiment and theory points to a particular set of hybrid functionals that provides an optimal description of this molecular system.

\section{Introduction}

Non-linear optical (NLO) materials have a non-linear response to the electric field associated with the light of a laser beam (i.e. the dielectric polarisation $\vec{P}$ responds non-linearly to the electric field $\vec{E}$ of the light), and this yields interesting phenomena such as second harmonic generation (SHG), third harmonic generation and difference-frequency generation. SHG, or frequency doubling, is a process in which two photons are combined to create a single photon with twice the original frequency, and it was discovered in 1961 by Franken, Hill, Peters

[a] Dr. P. S. P. da Silva, Prof. P. Martín-Ramos, Prof. M. Ramos Silva CFisUC, Department of Physics

University of Coimbra

Rua Larga 3004-516 Coimbra (Portugal)

E-mail:psidonio@fis.uc.pt

[b] Prof. P. Martín-Ramos

IUCA, University of Zaragoza, EPS

Carretera de Cuarte s/n 22071 Huesca (Spain)

[c] Dr. S. R. Domingos

Van 't Hoff Institute for Molecular Sciences

University of Amsterdam, Science Park 904

1098 HX Amsterdam (The Netherlands)

and

Current address:

Max Planck Institute for the Structure and Dynamics of Matter Luruper Chaussee 149, Geb.99.22761 Hamburg (Germany)

[d] M. d. C. Bota de Sousa, Dr. C. T. Arranja, Prof. A. J. F. N. Sobral CQC, Chemistry Department

University of Coimbra, Rua Larga 3004-535 Coimbra (Portugal)

$\square$ Supporting Information and the ORCID identification number(s) for the

(iD) author(s) of this article can be found under: https://doi.org/10.1002/cphc.201700975. and Weinreich ${ }^{[1]}$ at the University of Michigan. Since then many materials have been used for SHG, for example, lithium iodate $\left(\mathrm{LilO}_{3}\right)$, potassium niobate $\left(\mathrm{KNbO}_{3}\right)$ and potassium hydrogenphosphate $\left(\mathrm{KH}_{2} \mathrm{PO}_{4}\right)$. Besides the inorganic NLO materials, robust and durable organic molecules have also been considered for applications in this field, since they offer much larger flexibility in assembling different structures. Among the vast organic options, some molecules have donor and acceptor groups mediated by conjugated bonds and therefore show large hyperpolarisabilities. The dipole-dipole interactions between these dipolar molecules tend to aggregate them in antiparallel arrangements, which make the NLO response of the solid zero. Using molecules with a lambda shape may avoid such antiparallelism, since the molecules tend to stack on top of each other, as shown by Yamamoto, Katogi, Watanabe, Sato, Miyata and Hosomi, ${ }^{[2]}$ who achieved almost $90 \%$ non-centrosymmetric structures in a variety of space groups $(P 2, C C, C 2$, $14, c d)$.

Zyss $^{[3]}$ suggested the use of alternative molecules to build NLO solids: instead of dipolar $(A-\pi-D)$, octupolar structures $\left[(D-\pi-A)_{3}\right.$, where $D$ and $A$ refer to the electron-donating and electron-withdrawing groups, respectively] were proposed. Octupolar molecules have no static permanent dipole moment, and thus facilitate non-centrosymmetric crystallisation. Another advantage of these molecules is that the second-harmonic response does not depend on the polarisation of the incident light.

The critical step of crystallisation in a chiral group may be ensured by using co-formers with chiral centres, together with dipolar, octupolar or mixed-character molecules. ${ }^{[4,5]}$ Another strategy is to choose molecules that adopt a propeller-like 
shape and without a chiral centre assume a handedness that forces non-centrosymmetric assembly. ${ }^{[6]}$

Dicinnamalacetone (I) has an overall lambda shape with a propeller conformation, which caught our attention due to its potential as an SHG material. Before, its approximate $C_{2}$ symmetry had already been exploited to build lengthy molecules with liquid-crystal behaviour. ${ }^{[7]}$ Conformational flexibility influences the crystallisation processes with four possible outcomes: no crystallisation, crystallisation with several conformations disordered in the crystal, crystallisation with several conformations perfectly ordered in the crystal or crystallisation in several polymorphic forms. As will be shown below, I crystallises in a disordered structure. This structure and the associated optical properties were investigated by XRD and the KurtzPerry method. ${ }^{[8]}$

In computational chemistry, DFT has evolved into a mainstream method for the prediction of molecular properties over the past three decades. However, this has been accompanied by the appearance of a plethora of codes and density functional approximations and has raised the question about which one should be chosen, considering their differences in computation time and reliability of their results, bearing in mind the current controversy over whether good optimisations of energies may or may not reproduce good electron-density maps. ${ }^{[9-12]}$ Thus, the identification and pursuit of metrics and descriptors that can lead to an unbiased analysis is of utmost importance in this field. ${ }^{[12]}$

Hence, in addition to the experimental characterisation of I, calculations of the microscopic NLO properties were conducted with functionals from several families and with the secondorder Møller-Plesset perturbation method (MP2). To this end, excitation energies, oscillator strengths and transition dipole moments were estimated with 24 functionals/methods, which were compared in terms of their ability to determine these physical properties. Eight selected functionals/methods were further used to evaluate the macroscopic NLO properties of the solid compound by using the oriented-gas model.

\section{Results and Discussion}

\subsection{X-ray Structure Analysis}

The asymmetric unit contains one and a half independent molecules. The unit cell contains 16 such asymmetric units, that is, 24 full molecules of formula $\mathrm{C}_{21} \mathrm{H}_{18} \mathrm{O}$ (Table 1). The shape of the molecule resembles a butterfly, and in the solid state, the "wings", that is, the terminal phenyl groups, are not coplanar. Instead, they are oriented like the blades of a propeller, and thus the molecule becomes a chiral object, despite the absence of a carbon atom linked to four different groups. Both one and a half independent molecules are disordered over two conformations, so that four conformations are present in the crystal structure (see Figure 1). Each pair of conformations corresponds to one of the two helicities, as depicted in Figure 2.

The chiral crystal structure in space group F2dd is thus an enantiomeric mixture of the two conformational stereoisomers
Table 1. Crystal data and structure refinement.

\begin{tabular}{ll} 
empirical formula & $\mathrm{C}_{21} \mathrm{H}_{18} \mathrm{O}$ \\
formula weight & 286.35 \\
$T[\mathrm{~K}]$ & $293(3)$ \\
$\lambda[\AA]$ & 0.71073 \\
crystal system & orthorhombic \\
space group & $\mathrm{F} 2 \mathrm{dd}$ \\
$a[\AA]$ & $5.8085(2)$ \\
$b[\AA]$ & $33.1248(11)$ \\
$c[\AA]$ & $50.4824(16)$ \\
$V\left[\AA^{3}\right]$ & $9713.1(6)$ \\
$Z$ & 24 \\
$\rho[\mathrm{g}$ cm & $-3]$ \\
absorption coefficient $\left[\mathrm{mm}^{-1}\right]$ & 1.175 \\
$F(000)$ & 0.070 \\
crystal size [mm] & 3648 \\
$\theta$ range for data collection $\left[{ }^{\circ}\right]$ & $0.4 \times 0.08 \times 0.08$ \\
reflections collected & $1.6-25.6$ \\
independent reflections $[I>2 \sigma(I)]$ & 22244 \\
completeness to $2 \theta=51^{\circ}$ & 1782 \\
refinement method & $99.2 \%$ \\
data/restrains/parameters & full-matrix least-squares on $F^{2}$ \\
goodness of fit on $F^{2}$ & $4497 / 1 / 588$ \\
final $R$ index $[I>2 \sigma(I)]$ & 0.933 \\
$w R$ index (all data) & 0.0347 \\
largest diff. peak/hole & 0.0556 \\
& $0.087 /-0.115$ \\
\hline
\end{tabular}

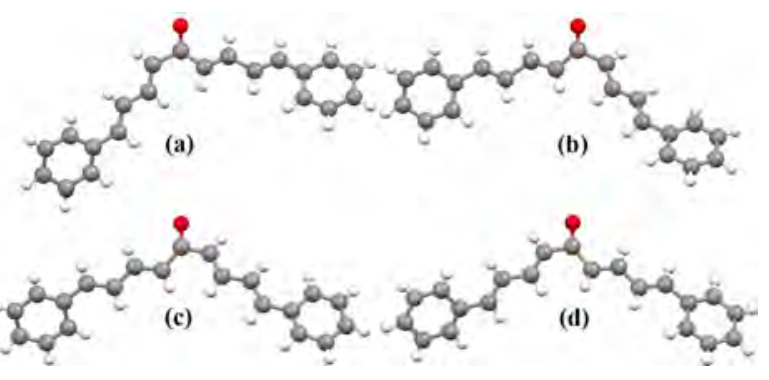

Figure 1. a) conformation 1, b) conformation 2, c) conformation 3, and (d) conformation 4.

(atropisomers) in a 2:1 ratio. The molecules do not establish conventional hydrogen bonds, due to the lack of donors. The molecules pack in columns, possibly due to $\pi \cdots \pi$ interactions between the phenyl rings. The centroid...centroid distances between neighbouring phenyl rings range from 4.70 to $4.98 \AA$. Figure 3 shows the crystal packing looking down from the top of the columns.

\subsection{Electronic Excitations}

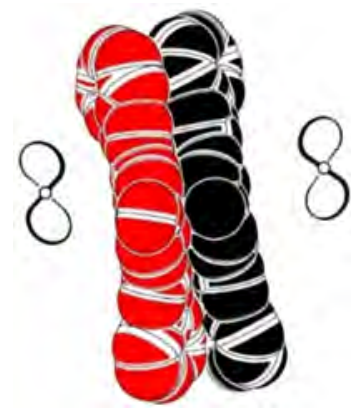

Figure 2. Conformations 1 and 2 are depicted in red; conformations 3 and 4 are depicted in black. Two helices are drawn to help visualisation of the molecular helicity.

Table 2 summarises the results of the calculations for the strongest singlet excitation of I with the solvent effects of dichloromethane described by the PCM model. The calculated properties 


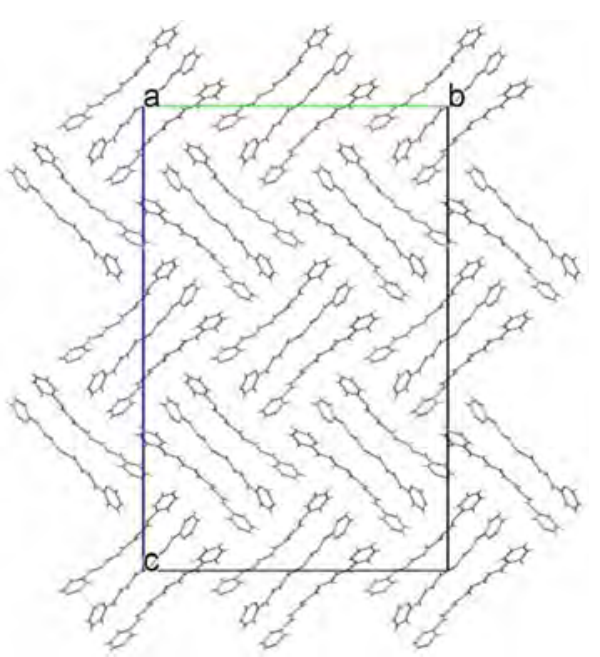

Figure 3. Crystal packing of the title compound. Only the major disordered component is depicted.

\begin{tabular}{|c|c|c|c|c|c|c|}
\hline Functional/method & Type $^{[a]}$ & $\chi$ & $\begin{array}{l}E \\
{[\mathrm{eV}]}\end{array}$ & $\mu_{\mathrm{ge}}[\mathrm{D}]$ & $f$ & $\Lambda$ \\
\hline SVWN5 $5^{[19,20]}$ & LDA & 0 & 2.388 & 10.518 & 1.002 & 0.725 \\
\hline BLYP[21,22] & GGA & 0 & 2.396 & 10.598 & 1.020 & 0.733 \\
\hline OLYP[23] & GGA & 0 & 2.425 & 10.534 & 1.020 & 0.732 \\
\hline $\mathrm{PBE}^{[24]}$ & GGA & 0 & 2.410 & 10.489 & 1.005 & 0.729 \\
\hline $\mathrm{M} 06-\mathrm{L}^{[25]}$ & mGGA & 0 & 2.587 & 10.689 & 1.121 & 0.733 \\
\hline TPSS $^{[26]}$ & mGGA & 0 & 2.484 & 10.670 & 1.072 & 0.731 \\
\hline $\operatorname{TPSSm}^{[27]}$ & mGGA & 0 & 2.484 & 10.653 & 1.069 & 0.731 \\
\hline $\operatorname{revTPSS}^{[28]}$ & $m G G A$ & 0 & 2.485 & 10.729 & 1.084 & 0.730 \\
\hline TPSSh $^{[29]}$ & $\begin{array}{l}\mathrm{GH}- \\
\mathrm{mGGA}\end{array}$ & 10 & 2.706 & 11.325 & 1.316 & 0.723 \\
\hline $\mathrm{M} 06^{[30]}$ & $\begin{array}{l}\mathrm{GH}- \\
\mathrm{mGGA}\end{array}$ & 27 & 2.917 & 12.078 & 1.613 & 0.719 \\
\hline $\mathrm{M} 08-\mathrm{HX}^{[31]}$ & $\begin{array}{l}\mathrm{GH}- \\
\mathrm{mGGA}\end{array}$ & 52.23 & 3.334 & 12.092 & 1.849 & 0.716 \\
\hline$M 06-2 X^{[30]}$ & $\begin{array}{l}\text { GH- } \\
\text { mGGA }\end{array}$ & 54 & 3.330 & 12.119 & 1.855 & 0.716 \\
\hline $\mathrm{M} 08-\mathrm{SO}^{[31]}$ & $\begin{array}{l}\text { GH- } \\
\text { mGGA }\end{array}$ & 56.79 & 3.298 & 12.229 & 1.870 & 0.716 \\
\hline $\mathrm{M} 06-\mathrm{HF}^{[32]}$ & $\begin{array}{l}\text { GH- } \\
\text { mGGA }\end{array}$ & 100 & 3.672 & 12.062 & 2.026 & 0.710 \\
\hline B3LYP[21, 22, 33] & GH-GGA & 20 & 2.831 & 11.784 & 1.491 & 0.719 \\
\hline$X 3 L_{Y P}^{[34]}$ & GH-GGA & 21.8 & 2.864 & 11.859 & 1.527 & 0.718 \\
\hline $\mathrm{PBEO}^{[35]}$ & GH-GGA & 25 & 2.947 & 11.913 & 1.586 & 0.718 \\
\hline BHHLYP[33] & GH-GGA & 50 & 3.330 & 12.513 & 1.978 & 0.717 \\
\hline LYP [22] & GH-GGA & 100 & 3.809 & 12.510 & 2.260 & 0.712 \\
\hline B2-PLYP ${ }^{[36]}$ & DH-GGA & 53 & 3.371 & 12.551 & 2.014 & 0.718 \\
\hline B2K-PLYP & DH-GGA & 72 & 3.588 & 12.622 & 2.168 & 0.721 \\
\hline CAM-B3LYP ${ }^{[38]}$ & RSH-GGA & $\begin{array}{l}19- \\
65\end{array}$ & 3.315 & 12.342 & 1.915 & 0.714 \\
\hline LC-BLYP[39] & RSH-GGA & $\begin{array}{l}0- \\
100\end{array}$ & 3.509 & 12.088 & 1.944 & 0.711 \\
\hline $\mathrm{TDHF}^{[40]}$ & WFT & 100 & 3.795 & 12.519 & 2.256 & 0.709 \\
\hline Experimental & & & 3.36 & & & \\
\hline
\end{tabular}

[a] GGA: generalised gradient approximation, mGGA: meta-GGA, GH: global hybrid, RSH: range-separated hybrid, $\mathrm{DH}$ : double hybrid, WFT: wave function theory.

are the vertical excitation energy $E$, the transition dipole $\mu_{\mathrm{ge}}$, the oscillator strength $f$, and a diagnostic quantity $\Lambda(0 \leq \Lambda \leq$
1), used to quantify the degree of spatial overlap between the occupied and virtual Kohn-Sham orbitals involved in the excitation. ${ }^{[13]}$ The $\Lambda$ diagnostic and other descriptors that measure orbital overlap ${ }^{[14,15]}$ are frequently used to describe the nature of the excitations and also to predict the performance of different functionals in TDDFT calculations. ${ }^{[16]}$ Small values of $\Lambda$ are associated with long-range excitations, and large values with short-range excitations. Usually, short-range excitations are expected to be described similarly by functionals with different amounts of Hartree-Fock (HF) exchange, whereas long-range excitations are expected to be poorly described by pure GGA functionals.

The strongest electronic excitation of $\mathbf{I}$ is dominated by a HOMO-LUMO transition and, as shown in Figure 4, there is a large overlap between these orbitals, leading to large $\Lambda$ values. According to Peach, Benfield, Helgaker and Tozer, ${ }^{[13]}$ for

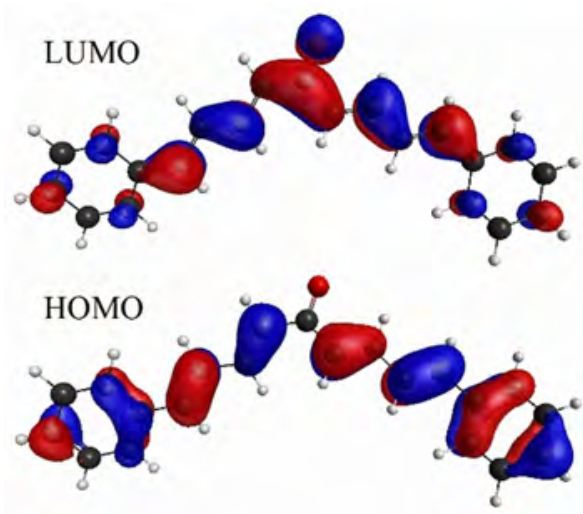

Figure 4. Frontier molecular orbitals of I obtained with the CAM-B3LYP functional (0.03 a.u. isodensity).

local excitations with large $\Lambda$ the performance of the functionals should be similar. Nonetheless, a difference of $1.413 \mathrm{eV}$ between the highest (LYP) and the lowest (BLYP) values of the excitation energy was observed in our study. However, several studies ${ }^{[17,18]}$ have pointed out that for $\pi$-conjugated systems the overlap of the canonical orbitals is not a good predictor of the functional performance in TDDFT calculations.

The excitation energy, oscillator strength and transition dipole increase with increasing percentage of HF exchange $\chi_{\text {, }}$ but stabilisation of the calculated transition dipole occurs around $50 \% \mathrm{HF}$ exchange (see Figure 5). The calculations that yield excitation energies closer to the experimental value (presented in the last row of Table 2), within the vertical approximation, are those obtained with the range-separated functional CAM-B3LYP and with the functionals with HF exchange near $50 \%$ : BHHLYP (50\%), B2-PLYP (53\%) and M06-2X (54\%).

It is interesting to compare our results with some recent articles on the benchmarking of calculations of excitation energies in conjugated systems. Jacquemin, Adamo, et al. have found in several studies ${ }^{[41-43]}$ that global hybrid functionals with around $25 \% \mathrm{HF}$ exchange are the most accurate for the calculation of vertical excitations. In studies on organic dyes, ${ }^{[44,45]}$ good performance of the CAM-B3LYP functional has been found in the 

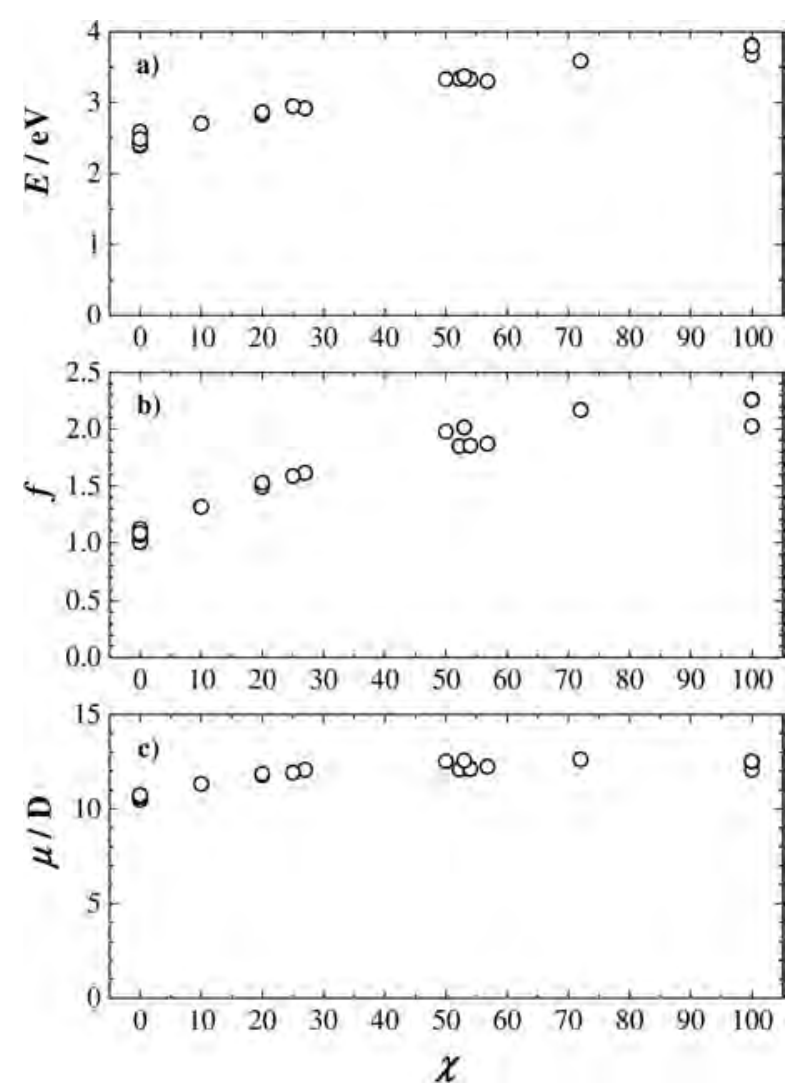

Figure 5. a) Excitation energy, b) oscillator strength and c) transition dipole moment versus the percentage of HF exchange $\chi$, for the strongest electronic excitation, with the solvent effects of dichloromethane described by the PCM model.

description of the excited states. Peach et al. have emphasised the high-quality excitations obtained with CAM-B3LYP, with a balanced description of all three categories of excitations (local, Rydberg and intramolecular charge transfer). ${ }^{[13,46]}$ The B2-PLYP double hybrid appears in a review of benchmark studies $^{[47]}$ as a star performer for the calculation of singlet excited states, with a mean absolute error smaller than $0.20 \mathrm{eV}$. Grimme et al. ${ }^{[48]}$ reached a similar conclusion, that is, B2-PLYP and B2GP-PLYP are more robust than hybrid functionals regardless of the system under study.

For a meaningful comparison with the experimental UV/Vis spectrum, the stick spectra obtained in the TDDFT calculations were convoluted with Gaussian functions with the appropriate full width at half-maximum (FWHM), typically $40 \mathrm{~nm}$; larger FWHM values were avoided, since they hide the weaker excitations. Figure 6 depicts the spectra obtained with the rangeseparated functional CAM-B3LYP and some representative functionals with different amounts of HF exchange: BLYP $(0 \%)$, M06-2X (54\%) and LYP (100\%).

The maximum of the BLYP spectrum is strongly red-shifted in comparison with the experimental one. This may be due to the limitations of TDDFT calculations with pure GGA functionals. This problem is usually found in calculations for chargetransfer systems and also for extended $\pi$-conjugated systems. The LYP spectrum is blue-shifted relative to the experimental

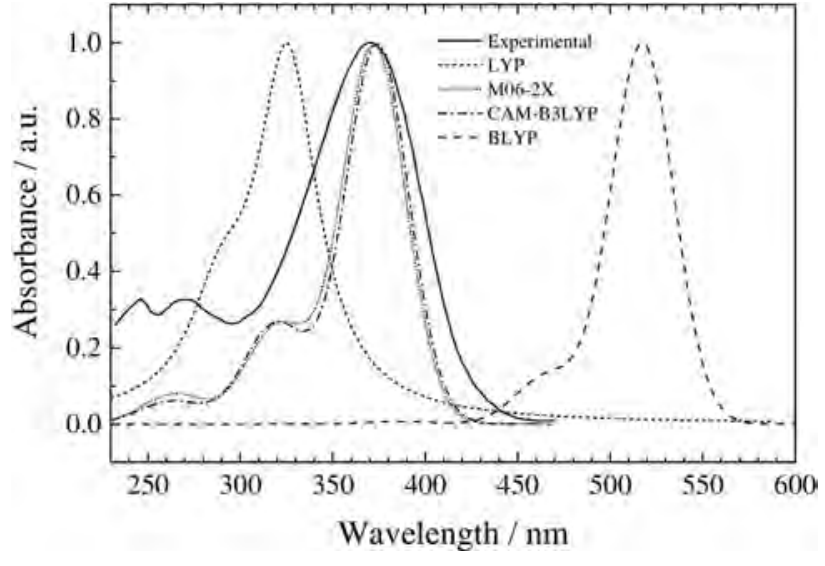

Figure 6. Comparison of the experimental and calculated (LYP, M06-2X, CAM-B3LYP, and BLYP) UV/Vis absorption spectra of I in dichloromethane.

one, while the CAM-B3LYP and M06-2X functionals resulted in the best agreement. As in the case of calculations with molecular charge-transfer systems, the introduction of a fixed amount of HF exchange in the hybrid functionals or the use of long-range-corrected functionals improved the theoretical results.

The Tamm-Dancoff approximation (TDA) ${ }^{[49]}$ is frequently used in the calculation of electronic excitations, since it is computationally less expensive than the full TDDFT model and the results have essentially the same quality. ${ }^{[50]}$ This approximation consists of neglecting the coupling between the excitations and de-excitations in the linear response TDDFT equation, reducing it to the symmetric eigenvalue problem [Eq. (1)]:

$A X^{\mathrm{TDA}}=\omega^{\mathrm{TDA}} X^{\mathrm{TDA}}$

where $A$ is the occupied-virtual block of the Hamiltonian matrix of the linear response TDDFT equation, $X^{\mathrm{TDA}}$ the excitation amplitudes (eigenvectors) and $\omega^{\mathrm{TDA}}$ the excitation energies (eigenvalues).

For negligible overlap between the pairs of occupied and virtual orbitals involved in an excitation, the TDDFT equations reduce to the TDA approximation.

According to Dreuw and Head-Gordon, ${ }^{[51]}$ the degree of charge transfer in an electronic transition can be assessed from the difference between the excitation energies obtained with the full TDDFT model and TDDFT in the TDA: the smaller the difference, the greater the charge-transfer character.

The electronic excited states were calculated in the gas phase and in dichloromethane by using the two aforementioned approaches. The results are presented in Table 3. As shown in Figure 7, the differences between the energies calculated with TDDFT and TDA methods are negligible for $0 \% \mathrm{HF}$ exchange but increase almost linearly with $\chi$. This effect appears both in the solvent and gas-phase calculations. For a large overlap of occupied and virtual orbitals, as in this case, a large difference between TDDFT and TDA is expected, but, apparently, this difference is also strongly dependent on the percentage of HF exchange of the functional used. 
Table 3. Theoretical energies, for the strongest electronic excitation, calculated with TDDFT and TDA in dichloromethane and in the gas phase.

\begin{tabular}{|c|c|c|c|c|c|c|c|}
\hline \multirow[t]{3}{*}{ Functional/method } & \multirow[t]{3}{*}{$\chi$} & \multicolumn{3}{|c|}{ Dichloromethane } & \multicolumn{3}{|c|}{ Gas phase } \\
\hline & & TDDFT & TDA & & TDDFT & TDA & \\
\hline & & $E[\mathrm{eV}]$ & $E[\mathrm{eV}]$ & $\Delta E[\mathrm{eV}]$ & $E[\mathrm{eV}]$ & $E[\mathrm{eV}]$ & $\Delta E[\mathrm{eV}]$ \\
\hline SVWN5 & 0 & 2.388 & 2.457 & 0.069 & 2.513 & 2.575 & 0.062 \\
\hline BLYP & 0 & 2.396 & 2.466 & 0.070 & 2.524 & 2.584 & 0.060 \\
\hline OLYP & 0 & 2.425 & 2.494 & 0.069 & 2.553 & 2.613 & 0.060 \\
\hline PBE & 0 & 2.410 & 2.478 & 0.068 & 2.536 & 2.597 & 0.061 \\
\hline M06-L & 0 & 2.587 & 2.653 & 0.066 & 2.731 & 2.790 & 0.059 \\
\hline TPSS & 0 & 2.484 & 2.553 & 0.069 & 2.620 & 2.681 & 0.061 \\
\hline TPSSm & 0 & 2.484 & 2.553 & 0.069 & 2.620 & 2.680 & 0.060 \\
\hline revTPSS & 0 & 2.485 & 2.554 & 0.069 & 2.623 & 2.684 & 0.061 \\
\hline TPSSh & 10 & 2.706 & 2.783 & 0.077 & 2.891 & 2.966 & 0.075 \\
\hline M06 & 27 & 2.917 & 3.008 & 0.091 & 3.155 & 3.257 & 0.102 \\
\hline M08-HX & 52.23 & 3.334 & 3.450 & 0.116 & 3.600 & 3.741 & 0.141 \\
\hline M06-2X & 54 & 3.330 & 3.446 & 0.116 & 3.598 & 3.740 & 0.142 \\
\hline M08-SO & 56.79 & 3.298 & 3.412 & 0.114 & 3.566 & 3.708 & 0.142 \\
\hline M06-HF & 100 & 3.672 & 3.871 & 0.199 & 3.945 & 4.157 & 0.212 \\
\hline B3LYP & 20 & 2.831 & 2.917 & 0.086 & 3.051 & 3.141 & 0.090 \\
\hline X3LYP & 21.8 & 2.864 & 2.951 & 0.087 & 3.091 & 3.183 & 0.092 \\
\hline PBE0 & 25 & 2.947 & 3.037 & 0.090 & 3.179 & 3.278 & 0.099 \\
\hline BHHLYP & 50 & 3.330 & 3.439 & 0.109 & 3.601 & 3.725 & 0.124 \\
\hline LYP & 100 & 3.809 & 3.995 & 0.186 & 4.074 & 4.275 & 0.201 \\
\hline B2-PLYP & 53 & 3.371 & 3.482 & 0.111 & 3.642 & 3.772 & 0.130 \\
\hline B2K-PLYP & 72 & 3.588 & 3.721 & 0.133 & 3.860 & 4.016 & 0.156 \\
\hline CAM-B3LYP & $19-65$ & 3.315 & 3.427 & 0.112 & 3.585 & 3.721 & 0.136 \\
\hline LC-BLYP & $0-100$ & 3.509 & 3.648 & 0.139 & 3.778 & 3.933 & 0.155 \\
\hline TDHF & 100 & 3.795 & 3.985 & 0.190 & 4.056 & $4.260^{(a)}$ & 0.204 \\
\hline $\mathrm{CIS}$ & & N/A & N/A & & 4.260 & N/A & \\
\hline
\end{tabular}

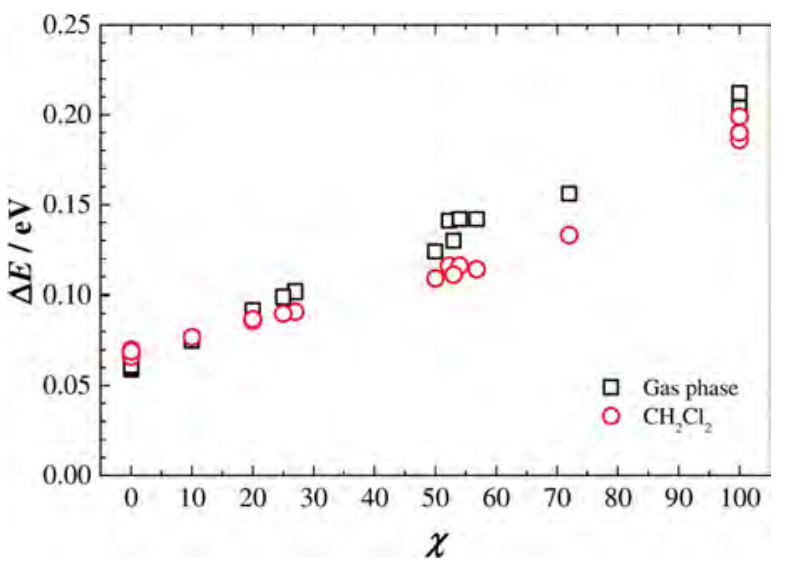

Figure 7. Plot of the energy differences for the strongest electronic excitation between results calculated with TDDFT and TDA methods in the gas phase (black squares) and in dichloromethane (red circles), as a function of the percentage of HF exchange $\chi$.

The calculated solvatochromic shifts are listed in Table 4. All calculations predict a bathochromic shift (red shift). This effect is exhibited by molecules that have a larger polarisation for the excited state than for the ground state. For this kind of molecules, polar solvents stabilise the excited state relative to the ground state, and this leads to a decrease in excitation energy. The absolute value of the solvatochromic shift increases with increasing amount of HF exchange but stabilises when this quantity reaches about $50 \%$ (see Figure 8 ).

\subsection{Non-linear Optical Properties}

\begin{tabular}{|llll|}
\hline \multicolumn{3}{|l}{ Table 4. Solvatochromic shifts calculated with TDDFT and TDDFT/TDA. } \\
Functional/method & $\chi$ & TDDFT & TDA \\
\hline & & $\Delta E^{\text {solv }}[\mathrm{eV}]$ & $\Delta E^{\text {solv }}[\mathrm{eV}]$ \\
SVWN5 & 0 & -0.125 & -0.118 \\
BLYP & 0 & -0.128 & -0.118 \\
OLYP & 0 & -0.128 & -0.119 \\
PBE & 0 & -0.126 & -0.119 \\
M06-L & 0 & -0.144 & -0.137 \\
TPSS & 0 & -0.136 & -0.128 \\
TPSSm & 0 & -0.136 & -0.127 \\
revTPSS & 0 & -0.138 & -0.130 \\
TPSSh & 10 & -0.185 & -0.183 \\
M06 & 27 & -0.238 & -0.249 \\
M08-HX & 52.23 & -0.266 & -0.291 \\
M06-2X & 54 & -0.268 & -0.294 \\
M08-SO & 56.79 & -0.268 & -0.296 \\
M06-HF & 100 & -0.273 & -0.286 \\
B3LYP & 20 & -0.220 & -0.224 \\
X3LYP & 21.8 & -0.227 & -0.232 \\
PBEO & 25 & -0.232 & -0.241 \\
BHHLYP & 50 & -0.271 & -0.286 \\
LYP & 100 & -0.265 & -0.280 \\
B2-PLYP & 53 & -0.271 & -0.290 \\
B2K-PLYP & 72 & -0.272 & -0.295 \\
CAM-B3LYP & $19-65$ & -0.270 & -0.294 \\
LC-BLYP & $0-100$ & -0.269 & -0.285 \\
TDHF & 100 & -0.261 & -0.275 \\
\hline
\end{tabular}




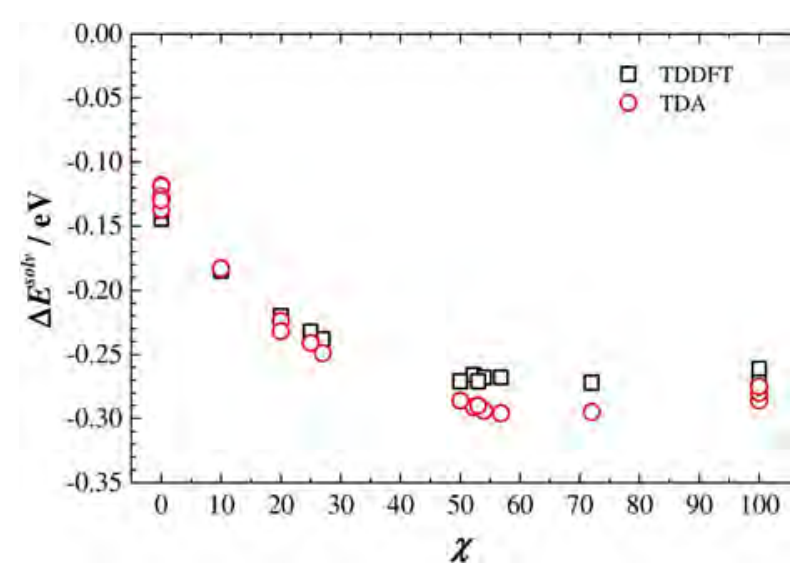

Figure 8. Plot of the solvatochromic shift for the strongest electronic excitation, calculated with TDDFT (black squares) and TDA (red circles) as a function of the percentage of HF exchange $\chi$.

\subsubsection{Experimental Kurtz and Perry Powder Results}

The Kurtz-Perry powder method ${ }^{[8]}$ was used to evaluate the NLO response of $\mathbf{I}$, and yielded an SHG efficiency of 0.7 times that of the urea standard in a first analysis with the grain sizes not standardised. This measure of the SHG efficiency has an uncertainty of about $10 \%$. With this technique, it is also possible to determine the phase-matching capabilities of a material. In a non-phase-matchable material, the maximum intensity is reached when the particle size approximately matches the average coherence length and then decreases with increasing particle size. In a phase-matchable material the intensity reaches a plateau at large particle sizes. To investigate the existence of phase matching in I, the dependence of intensity on particle size was monitored. The results ranged from 0.4 up to 1.3 times that of the urea standard but then decreased with increasing particle size (see Table S2 in the Supporting Information), and thus show that $\mathbf{I}$ is a non-phase-matchable material.

\subsubsection{Calculated Microscopic NLO Properties}

The $\beta_{i j k}$ tensor components were calculated for conformation 1 according to the methods described in the Experimental Section. The charge-transfer interaction occurs mainly in the $y x$ molecular plane (see Figure 9), and the dominant components of the first hyperpolarisability tensor are $\beta_{x x x}$ and $\beta_{x x y}$ The calculated values of the strongest components of the $\beta$ tensor are

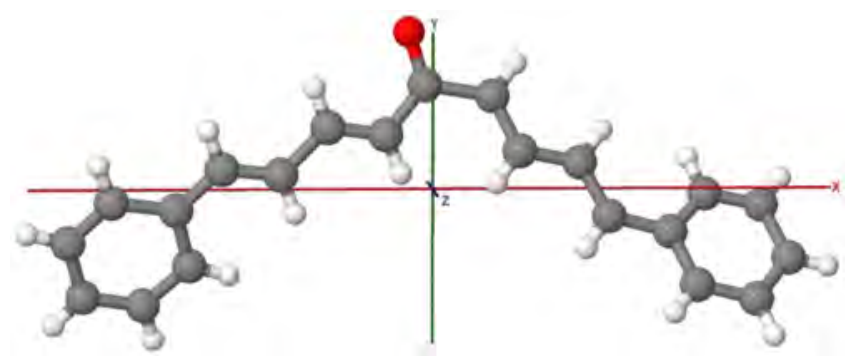

Figure 9. Molecular principal axes for conformation 1. listed in Table 5 for the different levels of theory used with indication of the type of functional/method and the percentage of HF exchange.

From the results presented in Table 5 and Figure 10, it is evident that a decrease of the absolute value of the hyperpolarisability components occurs with increasing percentage of HF exchange. The first hyperpolarisability is inversely proportional

\begin{tabular}{|c|c|c|c|c|}
\hline Functional/method & Type $^{[a]}$ & $\chi$ & $\beta_{x x x}$ & $\beta_{x x y}$ \\
\hline SVWN5 & LDA & 0 & 7693.1 & -5165.1 \\
\hline BLYP & GGA & 0 & 7706.5 & -5101.0 \\
\hline OLYP & GGA & 0 & 7524.8 & -5006.8 \\
\hline PBE & GGA & 0 & 7558.0 & -5060.8 \\
\hline M06-L & mGGA & 0 & 6548.1 & -4689.1 \\
\hline TPSS & mGGA & 0 & 7103.5 & -4932.7 \\
\hline TPSSm & mGGA & 0 & 7104.4 & -4929.3 \\
\hline revTPSS & mGGA & 0 & 7115.4 & -4963.1 \\
\hline TPSSh & GH-mGGA & 10 & 5559.5 & -4493.0 \\
\hline M06 & GH-mGGA & 27 & 3981.1 & -3908.6 \\
\hline BMK & GH-mGGA & 42 & 2761.5 & -3437.0 \\
\hline M08-HX & GH-mGGA & 52.23 & 2110.8 & -3166.5 \\
\hline M06-2X & GH-mGGA & 54 & 2070.3 & -3177.7 \\
\hline M08-SO & GH-mGGA & 56.79 & 1973.3 & -2919.9 \\
\hline M06-HF & GH-mGGA & 100 & 786.6 & -2283.9 \\
\hline B3LYP & GH-GGA & 20 & 4681.7 & -4217.5 \\
\hline X3LYP & GH-GGA & 21.8 & 4491.8 & -4149.3 \\
\hline PBEO & GH-GGA & 25 & 4051.1 & -3979.7 \\
\hline BHHLYP & GH-GGA & 50 & 2349.3 & -3118.7 \\
\hline LYP & GH-GGA & 100 & 991.3 & -1989.0 \\
\hline B2-PLYP & DH-GGA & 53 & 2169.8 & -2998.4 \\
\hline B2K-PLYP & DH-GGA & 72 & 1501.7 & -2499.8 \\
\hline CAM-B3LYP & RSH-GGA & $19-65$ & 2149.6 & -3182.4 \\
\hline LC-BLYP & RSH-GGA & $0-100$ & 1381.3 & -2794.6 \\
\hline $\mathrm{HF}$ & WFT & 100 & 981.9 & -1952.4 \\
\hline MP2 & WFT & 100 & 1290.8 & -3377.0 \\
\hline
\end{tabular}

[a] GGA: generalised gradient approximation, mGGA: meta-GGA, GH: global hybrid, $\mathrm{RSH}$ : range-separated hybrid, $\mathrm{DH}$ : double hybrid, WFT: wave function theory.

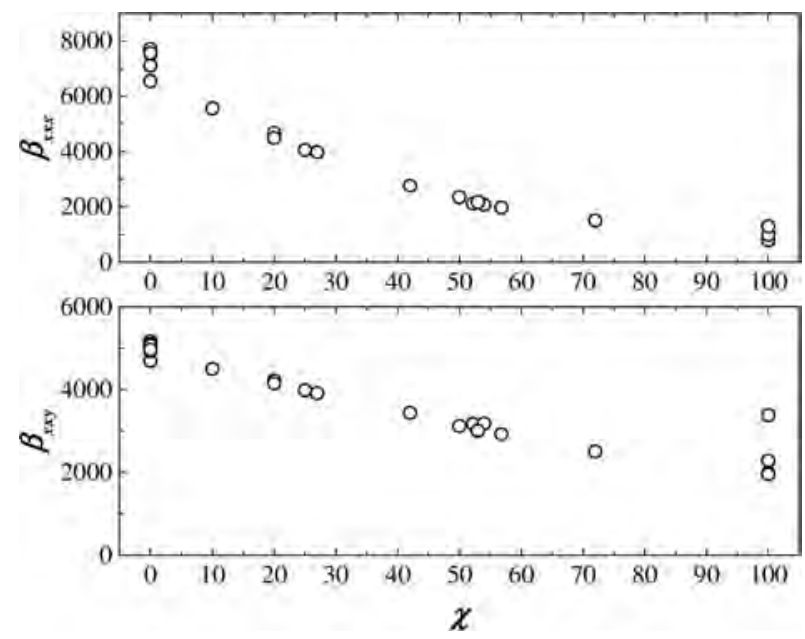

Figure 10. Plots of the absolute value of the two strongest static $\beta_{i j k}$ components for conformation 1 versus the percentage of HF exchange $\chi$. 
to the square of the excitation energies involved in multiphoton interactions and proportional to their respective oscillator strengths and transition dipoles, so a functional that describes correctly the molecular excitation processes should have also a good performance in the calculation of the $\beta$ tensor (the same reasoning applies for the polarisability $\alpha$ and for the second hyperpolarisability $\gamma$ ). To allow comparison with experiment, the macroscopic NLO properties were computed by using the calculated $\beta$ tensor.

\subsubsection{Calculated Macroscopic NLO Properties}

The crystal of $\mathbf{I}$ is orthorhombic in space group $F 2 d d$ and the polar point group is $m m 2$. Applying the Kleinman permutation symmetry, ${ }^{[52]}$ the non-vanishing independent components of the second-order susceptibility tensor allowed by this point group are $d_{z x x}, d_{z y y}$ and $d_{z z z}{ }^{[53]}$ The previously determined $\beta_{i j k}$ tensor components were used to calculate the macroscopic NLO coefficients $d_{i j k}$ by using the oriented-gas model with the Wortmann-Bishop local field factors. ${ }^{[54]}$

The SHG intensity $I^{\mathrm{HH}}$ is proportional to the square of the angular average of the susceptibility $\left\langle d^{2 \omega}\right\rangle$, calculated by using the expression deduced by Kurtz and Perry [Eq. (2)]: ${ }^{[8]}$

$$
\begin{aligned}
& I^{\mathrm{SHG}} \propto\left\langle d^{2 \omega}\right\rangle^{2}=\frac{19}{105} \sum_{i}\left(d_{i i i}^{2 \omega}\right)^{2}+\frac{13}{105} \sum_{i \neq j} d_{i i i}^{2 \omega} d_{i j j}^{2 \omega} \\
& +\frac{44}{105} \sum_{i \neq j}\left(d_{i i j}^{2 \omega}\right)^{2}+\frac{13}{105} \sum_{i j k, \mathrm{cyclic}} d_{i i j}^{2 \omega} d_{j k k}^{2 \omega}+\frac{5}{7}\left(d_{i j k}^{2 \omega}\right)^{2}
\end{aligned}
$$

The experimental result obtained with the Kurtz-Perry method is the ratio between the SHG intensities measured for $I$ and for the urea standard, so for a meaningful comparison between the computational and experimental results, calculations for urea with the same methodology as for the title compound were also performed.

The results of these calculations are listed in Table 6. The best agreement with the experimental value was obtained with the double hybrid B2-PLYP functional, the range-separated CAM-B3LYP functional and with M06-2X, a functional with $54 \%$ HF exchange, although the LYP, LC-BLYP and MP2 results were also reasonable. The hybrid functional with low HF exchange (B3LYP) and the pure GGA functional (BLYP) yielded values one order and two orders of magnitude larger than the experimental SHG result, respectively.

Previous studies on the calculation of NLO properties have shown that the range-separated functionals and hybrids with a high percentage of HF exchange (e.g. M06-2X) outperform both GGA functionals and global hybrids with low HF exchange. ${ }^{[55,56]}$ Champagne et al. studied 2-methyl-4-nitroaniline and concluded that double hybrids perform better in the determination of $\chi^{(2)}$, followed by the range-separated functionals CAM-B3LYP and LC-BLYP. ${ }^{[57]}$ A study on oligomers containing up to eight monomer units by Kirtman et al. ${ }^{[58]}$ found CAMB3LYP to be the most successful, amongst a set of exchangecorrelation functionals, for the calculation of several linear and non-linear optical properties.

In a thorough study on electro-optic chromophores with functionals of varying degrees of HF exchange, Johnson et al. ${ }^{[59]}$ concluded that the relationship between the percentage of long-range HF exchange and both $\beta$ (determined from experimental hyper-Rayleigh scattering) and $\lambda_{\max }$ is nearly linear, a trend similar to that reported in our study. Although none of the DFT methods examined by Johnson et al. offers small errors both in electronic excitations and hyperpolarisability, M06-2X and CAM-B3LYP came across as a good compromise.

Overall, when compared with the experimental values, both the absorption spectra and the macroscopic NLO properties, the best performance is achieved with the hybrid functionals with approximately $50 \%$ of exact exchange and with the range-separated functional CAM-B3LYP. Our findings do not seem to correlate with the conclusions of Medvedev, Bushmarinov, Sun, Perdew and Lyssenko, ${ }^{[9]}$ for whom a good functional must necessarily describe the charge density well. For these authors, a good functional is independent of the $\chi$ value: for instance, TPSS $(\chi=0 \%)$, PBE $(\chi=25 \%)$ and BHHLYP $(\chi=50 \%)$ are considered to describe the density well, while M06-L ( $\chi=$ $0 \%)$, M06 $(\chi=27 \%)$ and M06-2X $(\chi=54 \%)$ do not. In our study, of the six functionals mentioned above, only the functionals with $\chi$ around $50 \%$ performed well, regardless of the description of the density.

This does not imply that a correct description of electronic density is unimportant, but it cannot be deemed a sufficient condition to get good energies, a point already raised by Kepp. ${ }^{[10]}$ In his study, Kepp considers it questionable whether a very compact density regime is chemically relevant. Taking on

Table 6. Theoretical values of the macroscopic non-linear susceptibility components $\left[\mathrm{pm} \mathrm{V}^{-1}\right]$, angular average $\left\langle d^{2 \omega}\right\rangle$ and calculated SHG intensity ratio.

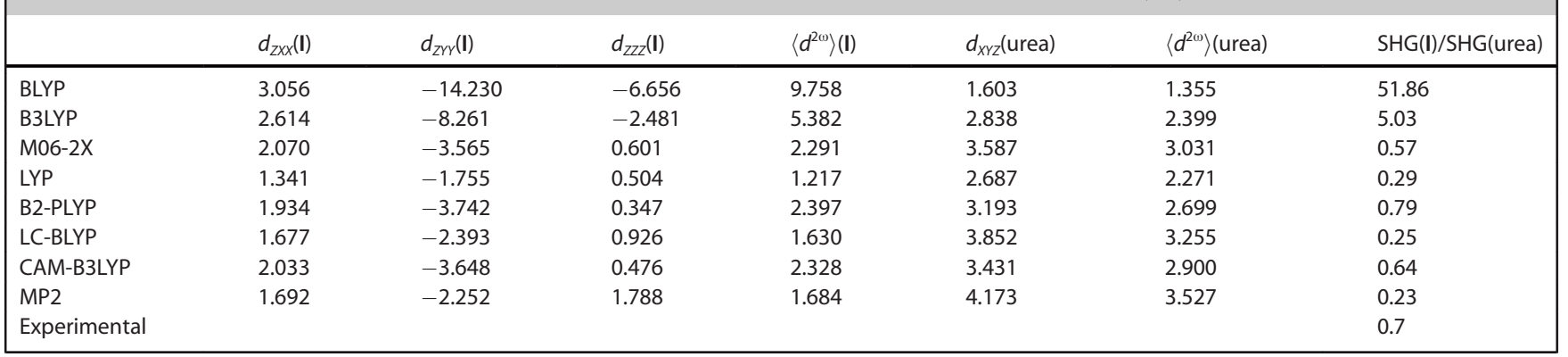


this controversy, Gould ${ }^{[12]}$ proposes the use of the more chemically relevant left Fukui functions to evaluate the quality of a functional, finding that optimised SOGGA11X, the hybrid-metaGGA TPSSh and double-hybrid B2PLYP can be regarded as star performers. Upon comparison of the performances of the functionals in this study and in aforementioned ones, no correlation between a good description of the energy and the ranking on the Fukui function could be found.

\section{Conclusions}

Four conformations were found to co-exist in the crystal structure of dicinnamalacetone in space group F2dd, which features an enantiomeric mixture with the two conformational stereoisomers in 2:1 ratio. The measured NLO response was on the same order of magnitude of that of urea. Regarding the computational chemistry calculations, hybrid functionals with $50 \%$ $\mathrm{HF}$, such as M06-2X, were observed to behave very well in terms of giving a good description of each of the physical properties in this work. In fact, stabilisation of the calculated transition dipoles or of the absolute value of the solvatochromic shift became evident around the aforementioned percentage of HF exchange. All things considered, the range-separated functional CAM-B3LYP showed the best performance both for the estimation of excitations and NLO properties in this study. Finally, apropos of the current controversy on whether good optimisations of energies may or may not reproduce good electron-density maps, the results obtained herein support Kepp's claim that a correct description of electronic density cannot be deemed a sufficient condition to get good energies, and no correlation between a good description of the energy and the ranking on the Fukui function could be found.

\section{Experimental Section}

\section{Materials and Synthesis}

Cinnamaldehyde (3-phenylprop-2-enal, [104-55-2], $\geq 95 \%$ ), acetone ([67-64-1], ACS reagent, $\geq 99.5 \%)$ and sodium hydroxide ([1310-732], reagent grade, $97 \%$, powder) were purchased from BDH Chemicals, Sigma Aldrich and JMGS Ltd., respectively.

Dicinnamalacetone $\quad[(1 E, 3 E, 6 E, 8 E)-1,9-$ diphenylnona-1,3,6,8-tetraen5-one] was synthesised by aldol condensation ${ }^{[60]}$ (see Figure 11): cinnamaldehyde $(4 \mathrm{~mL})$ was transferred to a $100 \mathrm{~mL}$ round-bottom flask, to which $\mathrm{NaOH}$ ( $15 \mathrm{~mL}$ of a $2 \mathrm{M}$ solution) was then added. Propanone was slowly added afterwards. The mixture was kept in a water bath at $30-35^{\circ} \mathrm{C}$ for $10-15$ min with vigorous stirring and an air condenser in the flask. The resulting solution was allowed to rest at room temperature for $30 \mathrm{~min}$ with occasional stirring. Upon cooling in ice water, the product crystallised. The crystals were col-

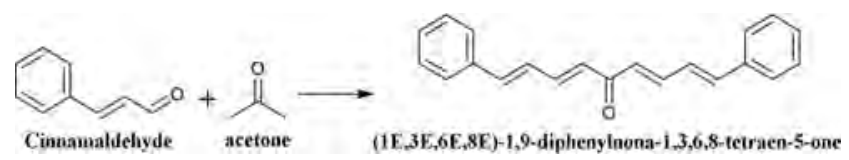

Figure 11. Synthesis of $(1 E, 3 E, 6 E, 8 E)$-1,9-diphenylnona-1,3,6,8-tetraen-5-one by aldol condensation. lected by suction filtration and washed with ice-cold water and then with an ice-cold $4 \%$ solution of acetic acid in ethanol. The reaction yield was $98 \%$.

For the purification, recrystallisation assays were conducted with heating in different solvents, and the largest crystals were obtained from ethanol/methanol $(1: 1 \mathrm{v} / \mathrm{v})$ solution. The melting point of the crystals, determined in a capillary, was $142-143^{\circ} \mathrm{C}$.

\section{X-ray Diffraction}

Diffraction data were collected at room temperature (293(2) K) by using graphite-monochromated $\mathrm{Mo}_{\mathrm{K \alpha}}$ radiation $(\lambda=0.71073 \AA$ ). Absorption corrections were made with SADABS. ${ }^{[61]}$ The structure was solved by direct methods with SHELXS-97 and refined anisotropically (non-H atoms) by full-matrix least-squares technique on $F^{2}$ by using the SHELXL-97 program. ${ }^{[62,63]}$ PLATON ${ }^{[64]}$ was used to analyse the structure and plot figures. Some disorder was observed, with each molecule occupying two close conformations, which were refined with an occupation close to $50 \%$.

CCDC 1563359 contains the supplementary crystallographic data for this paper. These data can be obtained free of charge from The Cambridge Crystallographic Data Centre.

\section{UV/Vis Absorption Spectroscopy}

The absorption spectrum was recorded with a Jasco V-530 doublebeam UV/Vis spectrophotometer in quartz cells with $1 \mathrm{~cm}$ path length with dichloromethane as solvent and reference.

\section{Kurtz-Perry Powder Method}

The SHG efficiency of I was measured by the Kurtz-Perry powder method. ${ }^{[8]}$ The measurements were performed at a wavelength of $1064 \mathrm{~nm}$ produced by a Nd:YAG laser operating at $10 \mathrm{~Hz}$ and producing $10 \mathrm{~ns}$ pulses with a pulse energy of $11 \mathrm{~mJ}$. For sample preparation, the material was ground to fine powder and then installed in the sample holder. In a first analysis, the sample grain sizes were not standardised. Signals between individual measurements varied in some cases by as much as $\pm 10 \%$. For a proper comparison with the urea reference material the measurements were averaged over several laser thermal cycles. To investigate the existence of phase matching in $\mathbf{I}$, one sample was ground to a fine powder and sieved into different grain size ranges $(<63,63-90,90-125,125-$ $180,180-212$ and $212-300 \mu \mathrm{m}$ ), and the thus-obtained six samples with different granularities were compacted and then installed in the sample holder. For a proper comparison with the urea reference material, the measurements were performed with urea sieved to the same grain size as the samples of $\mathrm{I}$.

\section{Computational Methods}

All ab initio and DFT calculations were performed with the GAMESS US package. ${ }^{[65]}$

\section{Geometry Optimisations}

The geometry optimisations of the four conformations found in the crystal structure were performed in the gas phase starting with the experimental $\mathrm{X}$-ray geometries. The calculations were performed by DFT with B3LYP for exchange and correlation, which combines the hybrid exchange functional of Becke ${ }^{[21,33]}$ with the 
correlation functional of Lee, Yang and Parr. ${ }^{[22]}$ The calculations were performed with an extended $6-311 \mathrm{G}(\mathrm{d}, \mathrm{p})$ basis set. At the end of each geometry optimisation a Hessian calculation was conducted to guarantee that the final structure corresponds to a true minimum, at the same level of theory as in the geometry optimisation. For the optimised geometries of the four different conformations, single-point energy calculations were performed with the conditions mentioned above (B3LYP functional and $6-311 \mathrm{G}(\mathrm{d}, \mathrm{p})$ basis set). Conformation 1 was also optimised by using the Polarizable Continuum Model (PCM) with the solvent dichloromethane, at the same level of theory as in previous calculations.

\section{Calculation of Electronic Excited States-Choice of Basis Set}

Prior to the calculation of the singlet excitations, with several different functionals, a study of the influence of the basis set on the excitation energies, transition dipoles and oscillator strengths was performed. The results for several Pople and Dunning-type correlation-consistent basis sets of increasing size are presented in the Supporting Information. From this analysis, the $6-311++G(d, p)$ basis set was chosen, since it gives a good compromise between the use of computational resources and convergence with the larger aug-cc-pVTZ basis set.

The electronic excited states of the optimised conformation 1 were calculated in the gas phase with the Configuration Interaction Singles (CIS) method and with time-dependent (TD) DFT. For the TDDFT calculations, the functionals listed below were used, with the exception of BMK, which is not supported for the calculation of electronic excitations in GAMESS US. These calculations were repeated with consideration of the solvent effect of dichloromethane by using the PCM. The calculation of the electronic excited states with solvent was not performed with the CIS method, since the solvent effects are not available for this method. The calculation of the electronic excitations was performed within the vertical approximation and a note of caution must be added, since experimental transitions are not purely vertical and the vibronic effects are often important. For example, Grimme et al. ${ }^{[48]}$ found differences between vertical and $0-0$ transitions ranging from 0.24 to $0.41 \mathrm{eV}$ for a set of five large molecules (regarding the difference between vertical and $0-0$ transitions see, for example, the review by Adamo and Jacquemin). ${ }^{[6]}$ However, the determination of coupling factors within the Franck-Condon approximation requires the calculation of the Hessian of the relevant excited state and this is, computationally, a very demanding task. This explains why the vast majority of TDDFT calculations uses the vertical approximation.

\section{Calculation of Microscopic Optical Properties}

To compare the relative performance of functionals from several different families, the static molecular linear polarisability $\alpha$ and static first hyperpolarisability $\beta$ tensor components were calculated only for conformation 1 with the following functionals:

1) Local density approximation (LDA) functional: SVWN5 (Slater exchange ${ }^{[19]}$ and VWN5 correlation). ${ }^{[20]}$

2) Parameter-free generalised gradient approximation (GGA) functionals: PBE (PBE exchange and correlation), ${ }^{[24]}$ BLYP (Becke exchange ${ }^{[21]}+$ LYP correlation$^{[22]}$ and OLYP (OPTX exchange ${ }^{[23]}+$ LYP correlation). ${ }^{[22]}$

3) Meta-GGA functionals: TPSS, ${ }^{[26]}$ TPSSm $^{[27]}$ (TPSS with modified parameter), revTPSS ${ }^{[28]}$ and M06-L, ${ }^{[25]}$ a local functional (no HF exchange) from the M06 family.
4) Global hybrid meta-GGA functionals: TPSSh $^{[29]}$ (TPSS $^{[26]}$ hybrid with $10 \% \mathrm{HF}$ exchange), $\mathrm{BMK}^{[67]}$ (42\% HF exchange) and, from the Minnesota family of functionals, $\mathrm{M}^{\left[{ }^{[30]}\right.}$ (27\% of HF exchange), M06-2X $\mathrm{X}^{[30]}$ (54\% HF exchange), M06- $\mathrm{HF}^{[32]}$ (100\% HF exchange), M08-HX $\mathrm{X}^{[31]}$ (52.23\% HF exchange) and $\mathrm{M}^{2} 8-\mathrm{SO}^{[31]}$ (56.79\% HF exchange).

5) Global hybrid GGA functionals: LYP ${ }^{[22]}$ (a pure correlation functional with $100 \% \mathrm{HF}$ exchange), $\mathrm{PBEO}^{[35]}$ ( $25 \% \mathrm{HF}$ exchange), BHHLYP ${ }^{[33]}$ (HF (50\%) and Becke exchange + LYP correlation), ${\text { B } 3 L_{Y P}{ }^{[21,22,33]} \text { (20\% HF exchange) and X3LYP }}^{[34]}(21.8 \% \mathrm{HF}$ exchange).

6) Double hybrid GGA functionals: B2-PLYP ${ }^{[36]}$ and B2K-PLYP. ${ }^{[37]}$ In these functionals, besides the substitution of some part of the GGA exchange by "exact" (HF) exchange, there is also mixing of GGA correlation with a non-local perturbative correlation energy obtained with a MP2-type treatment based on KohnSham orbitals and the corresponding eigenvalues. These functionals have two global parameters that describe the mixture of HF and Becke exchange $\left(a_{x}\right)$ and of MP2 and LYP correlation (c). For B2-PLYP, $a_{x}=53 \%, c=27 \%$, and for B2K-PLYP, $a_{x}=72 \%$, $c=42 \%$.

7) Range-separated functionals: LC-BLYP ${ }^{[39]}$ (long-range corrected version of the BLYP functional in which the amount of HF exchange increases continuously as a function of electron-electron separation distance) and CAM-B3LYP ${ }^{[38]}$ (Coulomb-attenuated B3LYP with 19\% HF plus $81 \%$ B88 exchange interaction at short range, and $65 \% \mathrm{HF}$ plus $35 \% \mathrm{~B} 88$ at long range, with the intermediate region smoothly described through the standard error function with parameter 0.33 ).

For comparison purposes, the $\alpha$ and $\beta$ tensors were also computed with the HF and MP2 methods. All calculations of microscopic optical properties were performed with the $6-311++G(d, p)$ basis set, and $\alpha$ and $\beta$ tensors were evaluated by finite field (FF) differentiation with an electric-field step of $f=0.001$ a.u. In these calculations, the input Cartesian coordinates were transformed into molecular principal axes (see Figure 9).

The $\alpha$ and $\beta$ tensor components of the four molecular conformations present in the asymmetric unit were also calculated with molecular axes equal to the crystal axes $(x=a, y=b, z=c)$. These tensors were then fed into the oriented gas model to calculate the macroscopic non-linear optical properties. The methods used were MP2 and some representative functionals of the above list with HF exchange ranging from 0 to $100 \%$ (BLYP, B3LYP, M06-2X, LYP) and the range-separated functionals CAM-B3LYP and LC-BLYP.

\section{Calculation of Macroscopic Optical Properties}

In most organic molecular crystals, it can be assumed that the intermolecular interactions are much weaker than the intramolecular chemical bonds. In this situation, the oriented gas model ${ }^{[68]}$ can be used to relate the macroscopic susceptibilities with the molecular hyperpolarisabilities. In this model, the crystalline susceptibilities are obtained by performing a tensor sum of the microscopic hyperpolarisabilities of the molecules within the unit cell [Eq. (3)]:

$$
\begin{aligned}
& d_{I J K}\left(\omega ; \omega_{1} ; \omega_{2}\right)= \\
& \frac{N}{V} f_{l}(\omega) f_{J}\left(\omega_{1}\right) f_{K}\left(\omega_{2}\right) \frac{1}{N_{\mathrm{g}}} \sum_{\mathrm{s}} \sum_{i j k} \cos \theta_{l i}^{(\mathrm{s})} \cos \theta_{j j}^{(\mathrm{s})} \cos \theta_{k k}^{(\mathrm{s})} \beta_{i j k}^{(\mathrm{s})}\left(\omega ; \omega_{1} ; \omega_{2}\right)
\end{aligned}
$$


where $I, J, K$ are the crystal axes, $N_{g}$ is the number of equivalent positions in the unit cell of volume $V$ having $N$ molecules, $f_{l}(\omega)$ are local field factors appropriate for the crystal axis $I$, and the cosine product terms represent the rotation from the molecular reference frame onto the crystal frame (these cosine terms are equal to 1 in the calculations presented herein, since a molecular axis equal to the crystal axis was used). The equivalent positions are labeled by the index $s$. The local field factors are essentially a correction for the difference between an applied field felt by the molecule in vacuum and the local field in the material.

In this work we used the Wortmann-Bishop ${ }^{[54]}$ model to calculate the local field factors. This model is an extension of Onsager's reaction field model ${ }^{[69]}$ and the details of this method can be found in ref. [4]. The Wortmann-Bishop local field factors are an improvement over the anisotropic Lorenz-Lorentz ${ }^{[70]}$ spherical cavity expression, widely used to calculate macroscopic NLO properties in crystals, but still falls short of the Rigorous Local Field derived by Munn and co-workers, ${ }^{[7]]}$ which is more accurate but computationally much more demanding.

\section{Acknowledgements}

The present work was financially supported by FCT Portugal with reference UID/FIS/04564/2016. The authors acknowledge the Laboratory for Advanced Computing at University of Coimbra (http://www.lca.uc.pt) for providing computing resources that have contributed to the research results reported within this paper. P.M-R would like to thank Santander Universidades for its financial support through the "Becas Iberoamérica Jóvenes Profesores e Investigadores, España 2017" scholarship program.

\section{Conflict of interest}

The authors declare no conflict of interest.

Keywords: atropisomerism • chirality · density functional calculations $\cdot$ nonlinear optics $\cdot$ solid-state structures

[1] P. A. Franken, A. E. Hill, C. W. Peters, G. Weinreich, Phys. Rev. Lett. 1961, 7, 118- 119.

[2] H. Yamamoto, S. Katogi, T. Watanabe, H. Sato, S. Miyata, T. Hosomi, Appl. Phys. Lett. 1992, 60, 935-937.

[3] J. Zyss, J. Chem. Phys.. 1993, 98, 6583-6599.

[4] P. S. P. Silva, C. U. Cardoso, M. R. Silva, J. A. Paixão, A. M. Beja, M. H. Garcia, N. Lopes, J. Phys. Chem. A 2010, 114, 2607-2617.

[5] S. R. Domingos, P. S. P. Silva, W. J. Buma, M. H. Garcia, N. C. Lopes, J. A Paixão, M. R. Silva, S. Woutersen, J. Chem. Phys. 2012, 136, 134501.

[6] V. Ostroverkhov, K. D. Singer, R. G. Petschek, J. Opt. Soc. Am. B 2001, 18, 1858.

[7] K. A. Hope-Ross, P. A. Heiney, J. F. Kadla, Can. J. Chem. 2010, 88, 639645.

[8] S. K. Kurtz, T. T. Perry, J. Appl. Phys. 1968, 39, 3798.

[9] M. G. Medvedev, I. S. Bushmarinov, J. Sun, J. P. Perdew, K. A. Lyssenko, Science 2017, 355, 49-52.

[10] K. P. Kepp, Science 2017, 356, 496.

[11] M. G. Medvedev, I. S. Bushmarinov, J. Sun, J. P. Perdew, K. A. Lyssenko, Science 2017, 355, 49-52.

[12] T. Gould, J. Chem. Theory Comput. 2017, 13, 2373-2377.

[13] M. J. G. Peach, P. Benfield, T. Helgaker, D. J. Tozer, J. Chem. Phys. 2008, 128,044118

[14] T. Le Bahers, C. Adamo, I. Ciofini, J. Chem. Theory Comput. 2011, 7, $2498-2506$.
[15] C. A. Guido, P. Cortona, B. Mennucci, C. Adamo, J. Chem. Theory Comput. 2013, 9, 3118-3126.

[16] P. Dev, S. Agrawal, N. J. English, J. Chem. Phys. 2012, 136, 224301.

[17] Z.-L. Cai, K. Sendt, J. R. Reimers, J. Chem. Phys. 2002, 117, 5543-5549.

[18] S. Grimme, M. Parac, ChemPhysChem 2003, 4, 292-295.

[19] J. C. Slater, Phys. Rev. 1951, 81, 385-390.

[20] S. H. Vosko, L. Wilk, M. Nusair, Can. J. Phys. 1980, 58, 1200-1211.

[21] A. D. Becke, Phys. Rev. A 1988, 38, 3098-3100.

[22] C. Lee, W. Yang, R. G. Parr, Phys. Rev. B 1988, 37, 785-789.

[23] N. C. Handy, A. J. Cohen, Mol. Phys. 2001, 99, 403-412.

[24] J. P. Perdew, K. Burke, M. Ernzerhof, Phys. Rev. Lett. 1996, 77, $3865-$ 3868.

[25] Y. Zhao, D. G. Truhlar, J. Chem. Phys. 2006, 125, 194101.

[26] J. Tao, J. Perdew, V. Staroverov, G. Scuseria, Phys. Rev. Lett. 2003, 91, 146401.

[27] J. P. Perdew, A. Ruzsinszky, G. I. Csonka, O. A. Vydrov, G. E. Scuseria, V. N. Staroverov, J. Tao, Phys. Rev. A 2007, 76, 040501.

[28] J. P. Perdew, A. Ruzsinszky, G. I. Csonka, L. A. Constantin, J. Sun, Phys. Rev. Lett. 2009, 103, 026403

[29] V. N. Staroverov, G. E. Scuseria, J. Tao, J. P. Perdew, J. Chem. Phys. 2003 $119,12129-12137$.

[30] Y. Zhao, D. G. Truhlar, Theor. Chem. Acc. 2008, 120, 215-241.

[31] Y. Zhao, D. G. Truhlar, J. Chem. Theory Comput. 2008, 4, 1849-1868.

[32] Y. Zhao, D. G. Truhlar, J. Phys. Chem. A. 2006, 110, 13126-13130.

[33] A. D. Becke, J. Chem. Phys. 1993, 98, 5648-5652.

[34] X. Xu, W. A. Goddard, Proc. Natl. Acad. Sci. USA 2004, 101, 2673-2677.

[35] C. Adamo, V. Barone, J. Chem. Phys. 1999, 110, 6158-6170.

[36] S. Grimme, J. Chem. Phys.. 2006, 124, 034108.

[37] A. Tarnopolsky, A. Karton, R. Sertchook, D. Vuzman, J. M. L. Martin, J. Phys. Chem. A 2008, 112, 3-8.

[38] T. Yanai, D. P. Tew, N. C. Handy, Chem. Phys. Lett. 2004, 393, 51-57.

[39] H. likura, T. Tsuneda, T. Yanai, K. Hirao, J. Chem. Phys.. 2001, 115, 3540 3544.

[40] A. D. McLachlan, M. A. Ball, Rev. Mod. Phys. 1964, 36, 844-855.

[41] D. Jacquemin, V. R. Wathelet, E. A. Perpète, C. Adamo, J. Chem. Theory Comput. 2009, 5, 2420-2435.

[42] D. Jacquemin, E. A. Perpète, I. Ciofini, C. Adamo, R. Valero, Y. Zhao, D. G. Truhlar, J. Chem. Theory Comput. 2010, 6, 2071-2085.

[43] D. Jacquemin, Y. Zhao, R. Valero, C. Adamo, I. Ciofini, D. G. Truhlar, J. Chem. Theory Comput. 2012, 8, 1255-1259.

[44] D. Jacquemin, E. A. Perpète, O. A. Vydrov, G. E. Scuseria, C. Adamo, J. Chem. Phys. 2007, 127, 094102.

[45] D. Jacquemin, E. A. Perpète, G. E. Scuseria, I. Ciofini, C. Adamo, J. Chem. Theory Comput. 2008, 4, 123-135.

[46] M. J. G. Peach, C. R. L. Sueur, K. Ruud, M. Guillaume, D. J. Tozer, Phys. Chem. Chem. Phys. 2009, 11, 4465.

[47] D. Jacquemin, B. Mennucci, C. Adamo, Phys. Chem. Chem. Phys. 2011, $13,16987$.

[48] L. Goerigk, J. Moellmann, S. Grimme, Phys. Chem. Chem. Phys. 2009, 11, 4611.

[49] S. Hirata, M. Head-Gordon, Chem. Phys. Lett. 1999, 314, 291 - 299.

[50] A. Chantzis, A. D. Laurent, C. Adamo, D. Jacquemin, J. Chem. Theory Comput. 2013, 9, 4517-4525.

[51] A. Dreuw, M. Head-Gordon, J. Am. Chem. Soc. 2004, 126, 4007-4016.

[52] D. A. Kleinman, Phys. Rev. 1962, 126, 1977-1979.

[53] R. W. Boyd, Nonlinear Optics, Academic Press, Burlington, 2008.

[54] R. Wortmann, D. M. Bishop, J. Chem. Phys. 1998, 108, 1001-1007.

[55] F. Castet, B. Champagne, J. Chem. Theory Comput. 2012, 8, 2044-2052.

[56] A. Plaquet, B. t. Champagne, L. Ducasse, E. Bogdan, F. Castet, AIP Conf Proc. 2015, 1642, $481-487$.

[57] T. Seidler, K. Stadnicka, B. Champagne, J. Chem. Theory Comput. 2014, 10, 2114-2124.

[58] I. W. Bulik, R. Zaleśny, W. Bartkowiak, J. M. Luis, B. Kirtman, G. E. Scuseria, A. Avramopoulos, H. Reis, M. G. Papadopoulos, J. Comput. Chem. 2013, $34,1775-1784$.

[59] L. E. Johnson, L. R. Dalton, B. H. Robinson, Acc. Chem. Res. 2014, 47, 3258-3265.

[60] T. J. Dickerson, T. Lovell, M. M. Meijler, L. Noodleman, K. D. Janda, J. Org. Chem. 2004, 69, 6603-6609.

[61] G. Sheldrick, SADABS, University of Göttingen, 1996.

[62] G. M. Sheldrick, Acta Crystallogr. Sect. A 2008, 64, 112-122. 
[63] G. M. Sheldrick, SHELXL97 and SHELXS97, University of Göttingen, 1997.

[64] A. L. Spek, J. Appl. Crystallogr. 2003, 36, 7-13.

[65] M. W. Schmidt, K. K. Baldridge, J. A. Boatz, S. T. Elbert, M. S. Gordon, J. H. Jensen, S. Koseki, N. Matsunaga, K. A. Nguyen, S. Su, T. L. Windus, M. Dupuis, J. A. Montgomery, J. Comput. Chem. 1993, 14, 1347-1363.

[66] C. Adamo, D. Jacquemin, Chem. Soc. Rev. 2013, 42, 845-856.

[67] A. D. Boese, J. M. L. Martin, J. Chem. Phys.. 2004, 121, 3405-3416.

[68] D. S. Chemla, J. L. Oudar, J. Jerphagnon, Phys. Rev. B 1975, 12, 45344546.
[69] L. Onsager, J. Am. Chem. Soc. 1936, 58, 1486-1493.

[70] J. Schwinger, L. L. DeRaad, Jr., K. Milton, W.-y. Tsai, J. Norton, Classical Electrodynamics, Perseus Books, Reading, MA, 1998.

[71] R. W. Munn, Mol. Phys. 2010, 89, 555-569.

Manuscript received: September 1, 2017

Revised manuscript received: November 14, 2017

Accepted manuscript online: November 14, 2017

Version of record online: December 13, 2017 